\title{
ARTICLE OPEN Thermal stability and electronic and magnetic properties of atomically thin 2D transition metal oxides
}

\author{
Heleen van Gog ${ }^{1}$, Wun-Fan $\mathrm{Li}^{1}$, Changming Fang ${ }^{2}$, Rik S. Koster ${ }^{1}$, Marjolein Dijkstra ${ }^{1}$ and Marijn van Huis (D)
}

Two-dimensional (2D) transition metal oxides (TMOs) are an emerging class of nanomaterials. Using density functional theory and ab initio molecular dynamics (AIMD) simulations, we carried out a systematic study of atomically thin metal oxide phases with compositions $\mathrm{MO}, \mathrm{M}_{2} \mathrm{O}_{3}$, and $\mathrm{MO}_{2}$, for transition metal elements $\mathrm{Sc}, \mathrm{Ti}, \mathrm{V}, \mathrm{Cr}$, and $\mathrm{Mn}$. We identified nine thermally stable structures that may be realized as free-standing nanosheets: hexagonal $h-\mathrm{Sc}_{2} \mathrm{O}_{3}, h-\mathrm{V}_{2} \mathrm{O}_{3}$, and $h-\mathrm{Mn}_{2} \mathrm{O}_{3}$; hexagonal $t$ - $\mathrm{VO}, t$ - $\mathrm{CrO}$, and $t-\mathrm{MnO}$; and square $s q-\mathrm{TiO}, s q-\mathrm{VO}$, and $s q-\mathrm{MnO}$. The $t-\mathrm{MO}$ phases are novel hexagonal structures which emerged naturally from phase transformations observed during AIMD simulations. The 2D TMOs were found to exhibit a wide range of remarkable electronic and magnetic properties, indicating that they are bright candidates for electronic and spintronic applications. Most exceptional in this regard is $h-\mathrm{V}_{2} \mathrm{O}_{3}$, that is the only phase that has been experimentally realized so far, and was found to be a ferromagnetic half-metal with Dirac-cone-like bands.

npj 2D Materials and Applications (2019)3:18; https://doi.org/10.1038/s41699-019-0100-z

\section{INTRODUCTION}

The discovery of graphene and its fascinating properties ${ }^{1}$ has incited a whole new field of research dedicated to the exploration of two-dimensional (2D) nanomaterials. Using top-down approaches, where monolayers are isolated from layered bulk structures, and bottom-up approaches, where thin films are obtained through deposition of molecules on substrates, added to graphene is now a wide range of $2 \mathrm{D}$ materials consisting of elements other than carbon, such as $h$-BN, the mono-elemental 2D semiconductors silicene, phosphorene, and germanene, Mxenes, i.e., transition metal carbides, nitrides, and carbonitrides, and transition metal dichalcogenides (TMDs), such as $\mathrm{MoS}_{2}{ }^{2,3}$ The exploration of 2D nanomaterials has only started little over a decade ago, but is rapidly progressing.

Bulk phase transition metal oxides (TMOs) are known to have unique properties originating from their itinerant and strongly correlated transition metal (TM) $d$ electrons. This strong electron correlation, where the TM $d$ electrons experience a strong Coulomb repulsion from one another, leads to physical phenomena such as the metal-insulator transition ${ }^{4,5}$ (MIT), where, upon tuning parameters such as temperature, pressure, and chemical composition, and often along with a structural and magnetic phase transition, the electronic structure of a system will undergo a MIT (for $\mathrm{VO}_{2}$, for example, see refs. ${ }^{6-8}$ ). In addition, TM $d$ orbitals can be either fully or partially occupied, giving rise to multivalence states of TM atoms and allowing numerous stoichiometries; vanadium oxide, for example, existing in the four principle phases $\mathrm{VO}, \mathrm{V}_{2} \mathrm{O}_{3}$, $\mathrm{VO}_{2}$, and $\mathrm{V}_{2} \mathrm{O}_{5}$, in the Wadsley phases ${ }^{9} \mathrm{~V}_{n} \mathrm{O}_{2 n+1}(1 \leq n \leq 3)$, and in the Magnéli phases ${ }^{10} \mathrm{~V}_{n} \mathrm{O}_{2 n-1}(3 \leq n \leq 9)$. A TMO can furthermore exist as various stable polymorphs, stoichiometrically identical but crystallographically different phases, that for $\mathrm{MnO}_{2}$, for example, add up to a total number of six stable phases found so far $^{11}$ and may transform into one another with energy barriers as low as
0.2-0.3 eV/f.u. ${ }^{12}$ Their diversity makes TMOs versatile in a wide range of applications, including energy storage, catalysis, molecular sieving, functional magnetic and optical materials, and biosensors.

With the popularity of $2 \mathrm{D}$ materials and the unique properties of bulk TMOs in mind, 2D TMOs are being actively investigated as well, and recently, the first thin film and nanosheet 2D TMOs have been realized experimentally. Limiting ourselves to the scope of our study, i.e., the early $3 d$ transition metal $(\mathrm{Sc}, \mathrm{Ti}, \mathrm{V}, \mathrm{Cr}$, and $\mathrm{Mn}$ ) oxides, and just naming a few works with no intention of a complete review: via chemical vapor deposition, Chang et al. prepared rutile $\mathrm{TiO}_{2}$ and monoclinic $\mathrm{VO}_{2}$ thin films on $a-\mathrm{Al}_{2} \mathrm{O}_{3}$ surfaces; ${ }^{13}$ with the same technique, epitaxial rutile and monoclinic $\mathrm{VO}_{2}$ films were grown on a $r-\mathrm{Al}_{2} \mathrm{O}_{3}$ substrate by Makarevich et al.; ${ }^{14}$ monolayered (ML) $\mathrm{V}_{2} \mathrm{O}_{3}$ thin films have been synthesized on a $\mathrm{Pd}(111)$ surface by Surnev at al. ${ }^{15,16}$ Exfoliation of a layered manganese oxide by Omomo et al. resulted in ML nanosheets of $\mathrm{MnO}_{2}$ in the T phase. ${ }^{17} \mathrm{~A}$ generalized approach to molecular selfassembly synthesis of ultrathin 2D nanosheets of transition metal oxides was developed by Sun et al., and resulted in the successful synthesis of, among others, $\sim 3.3$-nm-thick $\mathrm{TiO}_{2}$ nanosheets containing 4-5 MLs of anatase $\mathrm{TiO}_{2}{ }^{18}$ The preparation of 2D TMOs on temporary, water-soluble salt templates resulted in $\sim 1$ $\mathrm{nm}$-thick cubic MnO nanolayers and was reported by Xiao et al. ${ }^{19}$

Comprehensive computational studies using density functional theory (DFT) and the more advanced quasiparticle GW approach ${ }^{20}$ (G: Green's function; W: screened Coulomb interaction) on monolayered $\mathrm{MX}_{2}$ transition metal dichalcogenides and oxides (TMDs and TMOs) in the $\mathrm{T}$ and $\mathrm{H}$ phases were performed by Ataca et al. and Rasmussen et al., ${ }^{21,22}$ and included $\mathrm{ScO}_{2}$ and $\mathrm{MnO}_{2}$, which are stable in both phases, $\mathrm{TiO}_{2}$, which is unstable in either phase, and $\mathrm{VO}_{2}$ and $\mathrm{CrO}_{2}$, which only have a stable $\mathrm{H}$ phase. ${ }^{21}$ The monolayered $\mathrm{V}_{2} \mathrm{O}_{3}$ synthesized on a $\mathrm{Pd}(111)$ surface, mentioned in the previous paragraph, was characterized by DFT simulations by

\footnotetext{
${ }^{1}$ Soft Condensed Matter, Debye Institute for Nanomaterials Science, Utrecht University, Princetonplein 5, 3584 CC Utrecht, The Netherlands and ${ }^{2}$ Institute of Materials and Manufacturing, Brunel University London, Kingston Lane, Uxbridge, Middlesex UB8 3PH, UK
} Correspondence: Heleen van Gog (h.vangog@uu.nl) or Marijn van Huis (m.a.vanhuis@uu.nl)

Received: 11 January 2019 Accepted: 3 April 2019

Published online: 23 April 2019 
Kresse et al. ${ }^{23}$ DFT calculations by Vittadini et al. on 1-6 monolayer (ML) thick films derived from the main low-index surfaces of anatase $\mathrm{TiO}_{2}$ revealed two anomalously stable films that are a restructuring of anatase $\mathrm{TiO}_{2}$ into a lepidocrocite- $\mathrm{TiO}_{2}$ nanosheet (derived from the $2 \mathrm{ML}(001)$ film) and a novel $\mathrm{TiO}_{2}$ phase (derived from the $4 \mathrm{ML}$ (101) film). ${ }^{24}$ By DFT $+U^{25}$ (Hubbard $U$ parameter) and hybrid HSE06 ${ }^{26}$ (Heyd-Scuseria-Ernzerhof) calculations, Kan et al. identified the experimentally realized monolayered T-phase $\mathrm{MnO}_{2}$ nanosheets mentioned in the paragraph above to be an intrinsic ferromagnetic 2D material. ${ }^{27}$ Graphitic, hexagonal MnO layers were further predicted by means of DFT $+U$ by Kan et al. to be more stable than the corresponding wurtzite $w z-\mathrm{MnO}(0001)$ surface, when the number of layers is less than four. ${ }^{28}$ Along this line, Wang et al. performed DFT calculations and suggested that semiconductor $w z$ structures transform into planar hexagonal structures upon a planar stretching force. ${ }^{29}$

To the best of our knowledge, a systematic first-principles' study of single-atomically thin 2D TMO structures has not been conducted yet. Inspired by the successful synthesis of 2D hexagonal $\mathrm{V}_{2} \mathrm{O}_{3}$ on a substrate and the predicted stability of $2 \mathrm{D}$ hexagonal $\mathrm{MnO}$, we designed four different 2D TMO phases: hexagonal $\mathrm{MO}(h-\mathrm{MO})$ and square $\mathrm{MO}(s q-\mathrm{MO})$, which both have an equivalent bulk phase, namely wurtzite $\mathrm{MO}$ (wz-MO) and rocksalt $\mathrm{MO}$ ( $r s-\mathrm{MO})$, that can be built up directly from the $2 \mathrm{D}$ counterparts; and hexagonal $\mathrm{M}_{2} \mathrm{O}_{3}\left(h-\mathrm{M}_{2} \mathrm{O}_{3}\right)$ and square $\mathrm{MO}_{2}$ ( $s q$ $\mathrm{MO}_{2}$ ), for which no direct 2D bulk connection exists. Using planewave DFT and hybrid HSE06 calculations, we systematically disclosed the energetic and thermal stability, and novel electronic and magnetic properties, of these atomically thin 2D TMOs for the five early $3 d$ transition metals Sc, Ti, V, Cr, and Mn. Except for $h$ $\mathrm{V}_{2} \mathrm{O}_{3}$ and $h-\mathrm{MnO}$, all these structures are at this moment undiscovered.

\section{RESULTS AND DISCUSSION}

Computational methodology

The four planar $h-\mathrm{MO}, s q-\mathrm{MO}, h-\mathrm{M}_{2} \mathrm{O}_{3}$, and sq-MO input geometries considered in this study for the five $\mathrm{TMs}, \mathrm{Sc}, \mathrm{Ti}, \mathrm{V}$, $\mathrm{Cr}$, and $\mathrm{Mn}$, are shown in Fig. 1. For each of the 2D TMO input geometries, non-magnetic (NM), ferromagnetic (FM), and various anti-ferromagnetic (AFM) orderings of the TM atoms were investigated, including for $h$-MO, the ferrimagnetic (FiM) configuration that is the ground-state magnetic ordering of halfhydrated graphene (graphone). ${ }^{30}$ The number of (planar) structural-magnetic input configurations adds up to 20 for $h-\mathrm{MO}, 20$ for $s q-\mathrm{MO}, 15$ for $h-\mathrm{M}_{2} \mathrm{O}_{3}$, and 20 for $s q-\mathrm{MO}_{2}$. Inspired by the experimentally synthesized buckled structure of
$h-\mathrm{V}_{2} \mathrm{O}_{3},{ }^{15,16,23}$ we also examined the stability of buckled 2D TMO phases, rerunning all simulations with buckled instead of planar input geometries, with the O-sublayer shifted $0.5 \AA$ away from the TM-sublayer, bringing the total number of structural and magnetic input configurations considered in this study for the $h-\mathrm{MO}, s q-\mathrm{MO}$, $h-\mathrm{M}_{2} \mathrm{O}_{3}$, and $s q-\mathrm{MO}_{2}$ phases to 150 .

The DFT calculations were performed using the first principles' Vienna $A b$ initio Simulation Package ${ }^{31-33}$ (VASP). Within the projector-augmented wave (PAW) framework, ${ }^{34,35}$ the exchange and correlation energy terms were described using the Perdew-Burke-Ernzerhof (PBE) generalized gradient approximation (GGA) functional. ${ }^{36,37}$ Computational details are given in the Methods section. To obtain ground-state geometries and magnetic arrangements, the simulation cells were structurally and magnetically optimized, relaxing lattice parameters, atomic positions, and magnetic moments. To study the charges of the atoms, a Bader charge analysis ${ }^{38}$ was carried out using the approach by Henkelman et al. ${ }^{39,40}$

The enormous amount of calculations involved in our study prevented us from relaxing the structures with the more advanced Hartree-Fock (HF)/DFT hybrid Heyd-Scuseria-Ernzerhof functional $^{26}$ (HSE06). Previous studies have shown, however, that using HSE for 2D materials such as graphene halides ${ }^{41}$ and $\mathrm{MoS}_{2}{ }^{42}$ results in geometries that are almost identical to PBE-relaxed geometries. In addition, studies of bulk TMOs have found, at the same time, that, while giving very similar results for structural relaxation and relative stability and indicating that the nonlocal HF exchange has little effect on the structure and total energy of strongly correlated systems, the description of the electronic structure of such a system, in particular of its $d$ states, greatly improves when using a hybrid functional. ${ }^{1,43-47}$ To obtain a more accurate description of the electronic structure of our 2D TMOs, we therefore performed HSEO6 calculations on the PBE-relaxed structures that were found to be the 2D TMOs' structural and magnetic ground-state configurations. Employing the popular and computationally less expensive DFT $+U$ method was not considered in this study, as we are investigating a wide range of elements, and various physical properties such as energy, geometry, electronic structure, and magnetism, and the DFT $+U$ approach depends on the Hubbard $U$ parameter, the value of which differs for different elements and different properties, and may lead to unwanted inconsistencies in our results. ${ }^{44,48}$

To assess the thermal stability of the 2D TMOs, we performed ab initio molecular dynamics (AIMD) simulations for each of the 20 structural and magnetic ground-state configurations, using 72atom $6 \times 6$ supercells for the $h$-MO phases, 100 -atom $5 \times 5$ supercells for the sq-MO phases, 80 -atom $4 \times 4$ supercells for the (a) $h-\mathrm{MO}$
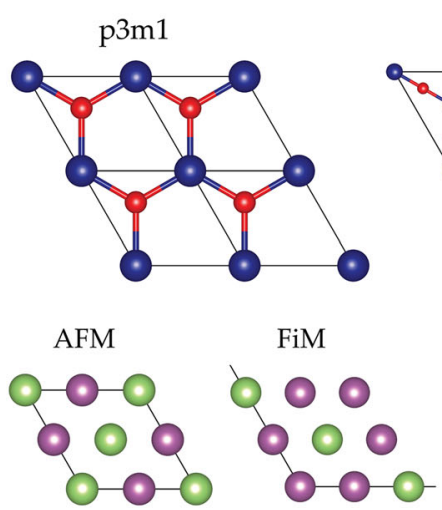

(b) $h-\mathrm{M}_{2} \mathrm{O}_{3}$
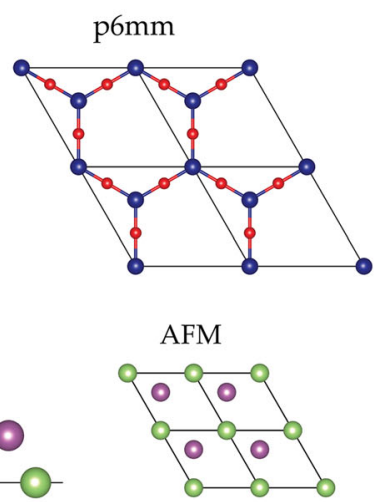

(c) $s q-\mathrm{MO}$

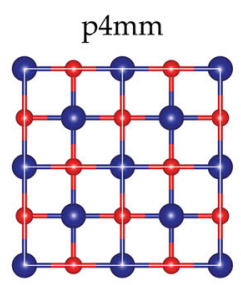

AFM1

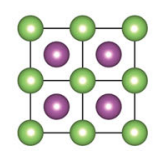

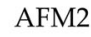

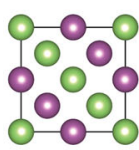

(d) $\mathrm{sq}-\mathrm{MO}_{2}$
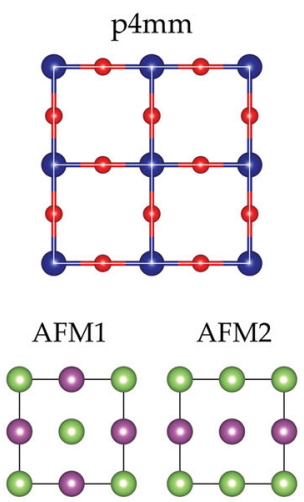

Fig. 1 Considered input geometries and anti-ferromagnetic orderings for the $h-\mathrm{MO}, s q-\mathrm{MO}, h-\mathrm{M}_{2} \mathrm{O}_{3}$, and $s q-\mathrm{MO}_{2}$ phases investigated in this study. Dark blue and red spheres denote TM and O atoms, respectively. Light green and purple spheres denote spin-up and spin-down TM atoms, respectively. Boundaries of simulation and unit cells are indicated with black or white solid lines 
Table 1. Summary of the structural, electronic, and magnetic properties, and thermal stability, of the structural and magnetic ground-state configurations for the 2D TMOs identified in this study

\begin{tabular}{|c|c|c|c|c|c|c|c|c|c|c|c|c|c|}
\hline $\begin{array}{l}\text { 2D } \\
\text { TMO }\end{array}$ & $\begin{array}{l}a \\
(\AA)\end{array}$ & $\begin{array}{l}\theta \\
\left({ }^{\circ}\right)\end{array}$ & $\begin{array}{l}E_{f} \\
\text { (eV/atom) }\end{array}$ & $\begin{array}{l}d_{b} \\
(\AA)\end{array}$ & EGS & MGS & $\begin{array}{l}E_{\mathrm{g}, \uparrow} \\
(\mathrm{eV})\end{array}$ & $\begin{array}{l}E_{\mathrm{g}, \downarrow} \\
(\mathrm{eV})\end{array}$ & $\begin{array}{l}m_{\mathrm{TM}} \\
\left(\mu_{B}\right)\end{array}$ & $\begin{array}{l}m_{\circ} \\
\left(\mu_{B}\right)\end{array}$ & $\begin{array}{l}q_{B, \mathrm{TM}} \\
(e)\end{array}$ & $\begin{array}{l}q_{B, O} \\
(e)\end{array}$ & Thermally stable? \\
\hline$t$-VO (a) & 5.423 & 119.5 & -1.335 & 0.44 & SC & AFM2 & 1.16 & 1.16 & \pm 2.27 & \pm 0.04 & 1.09 & -1.09 & Yes \\
\hline$t-\mathrm{MnO}(\mathrm{b})$ & $a \neq b^{\mathrm{a}}$ & 118.5 & -1.208 & - & SC & AFM1 & 1.69 & 1.69 & \pm 4.42 & \pm 0.07 & 1.30 & -1.30 & Yes \\
\hline$h-\mathrm{ScO}$ & 3.774 & 60.4 & -2.367 & 0.53 & SC & AFM & 1.05 & 1.05 & \pm 0.21 & \pm 0.02 & 1.41 & -1.41 & No \\
\hline$h$-TiO & 3.092 & 60.0 & -1.753 & 0.71 & SC & NM & 0.28 & - & - & - & 1.27 & -1.27 & No \\
\hline$h-\mathrm{CrO}$ & 3.379 & 52.3 & -1.038 & - & M & AFM & - & - & \pm 3.66 & \pm 0.08 & 1.32 & -1.32 & No \\
\hline$h-\mathrm{MnO}$ & 3.282 & 64.2 & -1.137 & - & SC & AFM & 2.11 & 2.11 & \pm 4.61 & \pm 0.08 & 1.42 & -1.42 & No \\
\hline$s q-\mathrm{ScO}$ & 4.221 & 90.0 & -2.470 & - & M & NM & - & - & - & - & 1.62 & -1.62 & No \\
\hline$s q-\mathrm{TiO}$ & 4.038 & 90.0 & -1.942 & - & M & AFM1 & - & - & \pm 1.22 & 0.00 & 1.50 & -1.50 & Yes \\
\hline$s q-\mathrm{VO}$ & 3.973 & 90.0 & -1.430 & - & SC & AFM1 & 3.12 & 3.12 & \pm 3.66 & 0.00 & 1.46 & -1.46 & Yes \\
\hline$h-\mathrm{V}_{2} \mathrm{O}_{3}$ & 6.181 & 60.0 & -1.821 & - & $\mathrm{HM}$ & $\mathrm{FM}$ & - & 6.24 & 2.10 & -0.06 & 1.65 & -1.10 & Yes \\
\hline$h-\mathrm{Cr}_{2} \mathrm{O}_{3}$ & 6.137 & 60.0 & -1.394 & - & SC & $\mathrm{FM}$ & 0.88 & 5.73 & 2.93 & 0.05 & 1.65 & -1.10 & No \\
\hline$h-\mathrm{Mn}_{2} \mathrm{O}_{3}$ & 6.159 & 60.0 & -1.225 & - & SC & FiM & 3.02 & 3.77 & $\mathrm{FiM}^{\mathrm{b}}$ & 0.05 & 1.44 & -1.02 & Yes \\
\hline$s q-\mathrm{ScO}_{2}$ & 4.008 & 90.0 & -1.980 & - & $\mathrm{HM}$ & $\mathrm{FM}$ & 5.21 & - & -0.09 & 0.54 & 2.08 & -1.04 & No \\
\hline$s q-\mathrm{TiO}_{2}$ & 3.746 & 90.0 & -2.144 & - & SC & NM & 0.13 & - & - & - & 2.28 & -1.14 & No \\
\hline$s q-\mathrm{VO}_{2}$ & 3.647 & 90.0 & -1.681 & - & SC & $\mathrm{FM}$ & 0.65 & 1.93 & 1.12 & -0.06 & 2.06 & -1.03 & No \\
\hline$s q-\mathrm{CrO}_{2}$ & 3.580 & 90.0 & -1.378 & - & $\mathrm{HM}$ & $\mathrm{FM}$ & - & 2.91 & 2.54 & -0.27 & 1.93 & -0.97 & No \\
\hline$s q-\mathrm{MnO}_{2}$ & 3.556 & 90.0 & -1.114 & - & $\mathrm{HM}$ & AFM1 & 4.07 & - & \pm 3.48 & 0.50 & 1.93 & -0.96 & No \\
\hline
\end{tabular}

$h-\mathrm{M}_{2} \mathrm{O}_{3}$ phases, and 72 -atom $5 \times 5$ supercells for each of the $s q$ $\mathrm{MO}_{2}$ phases, except $s q-\mathrm{MnO}_{2}$, which was simulated using a 108atom $6 \times 6$ supercell to accommodate its magnetic ordering. For each of the structures, with a time step of $1 \mathrm{fs}$, a 2 -ps initial equilibration and a 5 -ps subsequent canonical (NVT) ensemble simulation using a Nosé thermostat ${ }^{49-51}$ was carried out, at simulation temperatures of 300 and $700 \mathrm{~K}$. After the AIMD simulations, AIMD supercell output configurations were relaxed at high accuracy at $0 \mathrm{~K}$ again, to see if the structures had remained topologically equivalent.

Energetic stability of the $h-\mathrm{MO}, s q-\mathrm{MO}, h-\mathrm{M}_{2} \mathrm{O}_{3}$, and $s q-\mathrm{MO}_{2}$ phases

To assess the relative stability of the $2 \mathrm{D}$ TMO phases, we define their formation energy per atom, $E_{f}$, relative to their elemental transition metal bulk phases TM and the paramagnetic oxygen molecule $\mathrm{O}_{2}$ :

$E_{f}=\frac{1}{1+x}\left[E\left(\mathrm{MO}_{x}\right)-E(\mathrm{TM})-\frac{x}{2} E\left(\mathrm{O}_{2}\right)\right]$,

where $E\left(\mathrm{MO}_{x}\right)$ is the calculated total energy of a $2 \mathrm{D}$ TMO with $x=1,1.5$, or $2 ; E(T M)$ is the energy per atom of the TMs in their elemental phase; and $E\left(\mathrm{O}_{2}\right)$ is the energy of an $\mathrm{O}_{2}$ molecule in its paramagnetic ground state.

Table S1 in the Supplementary Information (SI) lists the calculated energies of lowest-energy and higher-energy magnetic and nonmagnetic configurations for all 2D TMO phases considered in this study. This table includes $t$-MO phases, which were found from the AIMD simulations and which will be discussed separately below. A summary of the formation energy $E_{f}$ of the most stable magnetic ground-state configurations can be found in Table 1. For the latter, a concave-hull plot is included as well (see Fig. 2). In general, a system will be unstable and decompose into its two neighboring compositions if its energy lies above the concave-hull line, i.e., the common tangent constructed connecting line. In the concave-hull plot for the 2D TMOs, for each of the phases the formation energy is located below the straight line connecting the two adjacent data points. This suggests that all considered 2D TMO phases are energetically stable at the respective stoichiometries.

Comparing the formation energies of the $h-\mathrm{MO}$ and $s q-\mathrm{MO}$ polymorphs, the sq-MO phase was found to be energetically more favorable than the $h-\mathrm{MO}$ phase for all considered TMs except Mn, sq-MnO being less stable than $h-\mathrm{MnO}$ by $39 \mathrm{meV} / \mathrm{atom}$. The relative stability of the $s q-\mathrm{MO}$ phases over $h-\mathrm{MO}$ may be due to the bonding network, for which sq-MO, where each TM atom is coordinated by four oxygen atoms, resembles that of its $3 \mathrm{D} r \mathrm{rs}$ counterpart. In general, rs compounds have a stronger ionic nature than corresponding $w z$ phases. The stronger ionicity of the $\mathrm{TM}-\mathrm{O}$ bonds in sq-MOs is indeed evidenced by the Bader charge analysis of the 2D structures: as can be seen from Table 1, for all TMs except Mn, the TM atoms were found to have a Bader charge that is $\sim 0.2 e$ higher in the $s q-\mathrm{MO}$ phase than in the corresponding $h$-MO phase; for $\mathrm{MnO}$, the Bader charge on the $\mathrm{Mn}$ atoms was 


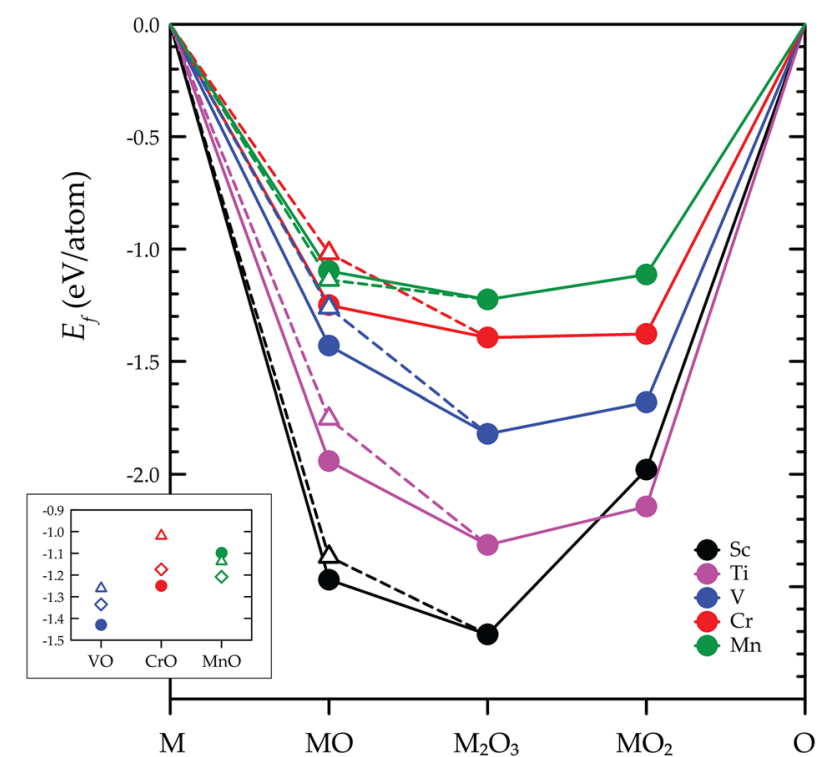

Fig. 2 Concave-hull plot of the formation energies $E_{f}$ of the $h$-MO phases (triangle markers), and $s q-\mathrm{MO}, h-\mathrm{M}_{2} \mathrm{O}_{3}$, and $s q-\mathrm{MO}_{2}$ phases (circle markers). The inset shows the formation energies of the three $t$-MO phases (diamond markers) and their corresponding $h$-MO (triangle markers) and sq-MO (circle markers) phases. The formation energies are also listed in Table 1

found to be similar in magnitude in both phases. TM and $\mathrm{O}$ atom coordination numbers and TM-O bond lengths in the 2D TMOs (listed in Table S2) can be correlated with the calculated atomic Bader charges (listed in Table 1): In sq-MO, the coordination number of all atoms is 4 , whereas for $h$-MO this number is 3 . The larger number of bonds correlates with an increased ionicity for sq-MO structures, while at the same time the bond lengths in the latter are slightly larger than those in corresponding $h$-MO structures. Comparing $\mathrm{MO}, \mathrm{M}_{2} \mathrm{O}_{3}$, and $\mathrm{MO}_{2}$ structures, it can be observed that the Bader charge on the TM atoms is dominated by the difference in stoichiometry, and increases when going from $\mathrm{MO}$ to $\mathrm{M}_{2} \mathrm{O}_{3}$ to $\mathrm{MO}_{2}$. For the latter two structures, the bond lengths are considerably smaller than those in $h-\mathrm{MO}$ and sq-MO phases, by up to $10 \%$, which is likely related to the fact that the $O$ atoms have only two TM nearest neighbor atoms in $\mathrm{M}_{2} \mathrm{O}_{3}$ and $\mathrm{MO}_{2}$.

For further analysis of the structural buckling, we define the buckling energy $E_{b}$ as the energy difference between a $d_{b}$ buckled structure and its planar counterpart:

$E_{b}=E\left(\mathrm{TMO}_{\text {buckled }}\right)-E\left(\mathrm{TMO}_{\text {planar }}\right)$,

where a negative $E_{b}$ indicates that a buckled structure is energetically more stable than the corresponding planar structure, and $d_{b}$ is simply the perpendicular separation between the TMand O-sublayer.

Relaxation of the 150 input configurations for the $h-\mathrm{MO}$, sq-MO, $h-\mathrm{M}_{2} \mathrm{O}_{3}$, and $s q-\mathrm{MO}_{2}$ phases resulted in 24 stable and metastable buckled structures. Of these, 15 were found to have $\left|E_{b}\right| \leq 20 \mathrm{meV} /$ atom and $d_{b} \geq 0.02 \AA$ (see Table S1), implying that these 2D TMO phases have a rather flat potential energy surface (PES), where the energies of planar and buckled geometries are two nearby local minima. Five buckled structures, namely AFM $h$-ScO, NM $h$-TiO, FM $h$-VO, AFM1 sq-CrO, and AFM $h-\mathrm{Ti}_{2} \mathrm{O}_{3}$, were found to be structural and magnetic ground-state configurations, with buckling ranging from 0.14 to $0.71 \AA$ (see Table 1). The reason for the energetic stability of these buckled 2D phases may be the pseudoJahn-Teller effect ${ }^{52}$ (PJTE), which states that if the excited-state geometry of a polyatomic system is very close in energy (nearly degenerate) to its ground-state geometry, then these two states will be mixed via vibronic coupling, resulting in a symmetrybreaking distortion which stabilizes the system. The PJTE has been commonly seen in other 2D systems, such as group-IV ${ }^{53,54}$ and group $-\mathrm{V}^{55}$ nanosheets.

\section{Energetic stability with respect to bulk TMOs}

The stability of the $h-\mathrm{MO}, s q-\mathrm{MO}, h-\mathrm{M}_{2} \mathrm{O}_{3}$, and $s q-\mathrm{MO}_{2}$ phases with respect to their $3 \mathrm{D}$ counterparts is evaluated by comparing their total energies per atom directly:

$\Delta E=E(2 \mathrm{D})-E(3 \mathrm{D})$.

This simple formula has been used to evaluate the stability of cadmium chalcogenide monolayers ${ }^{56}$ and other 2D materials ${ }^{57}$ with respect to their bulk counterparts. A negative $\Delta E$ means that a 2D monolayer is energetically more favorable than its corresponding $3 \mathrm{D}$ bulk phase, indicating that a material would grow into a 2D slab rather than into a 3D nanocrystal. Furthermore, Singh et al. calculated the formation energies of a range of $2 \mathrm{D} \mathrm{ML}$ materials, and came to the conclusion that all $2 \mathrm{D}$ materials that have been synthesized experimentally in the freestanding form, have a $\Delta E$ smaller than $200 \mathrm{meV} /$ atom, ${ }^{58}$ suggesting that this could be a first selection criterion for assessing stability.

When choosing the 2D TMOs' corresponding 3D phases, in addition to having the same stoichiometry as the $2 \mathrm{D}$ phases, two criteria were applied to make the chosen bulk structures as relevant as possible: (1) bulk structures that can be built up directly from the 2D counterparts are preferred. This is the case for the 3D rs-MO and $w z-\mathrm{MO}$ phases, which can be built up directly from the 2D sq-MO and $h-\mathrm{MO}$ phases. (2) Bulk structures that are relatively stable at low temperatures are preferred. This is to avoid possible structural and magnetic phase transitions at higher temperatures; and it makes more sense to compare lower energy structures rather than higher-energy structures with the 2D phases, which were calculated at $0 \mathrm{~K}$.

The phases that were selected as the 2D TMOs' corresponding bulk phases are listed in Table S2. Five bulk phases that have not been experimentally realized yet and remain hypothetical were still chosen because of their close similarity to the 2D TMO phases. The structural and magnetic ground states for these phases, i.e., $w z-\mathrm{ScO}, w z-\mathrm{TiO}, w z-\mathrm{VO}, w z-\mathrm{CrO}$, and $r s-\mathrm{ScO}$, were calculated and can be found in Table S2. The magnetic ground state for $r s-\mathrm{CrO}$ and bixbyite $\mathrm{Sc}_{2} \mathrm{O}_{3}$, whose magnetic orderings were not found in literature, was investigated as well. No bulk phase was chosen for $\mathrm{ScO}_{2}: \mathrm{ScO}_{2}$ has neither been experimentally realized nor theoretically predicted, and there is not a clear structural connection between the $3 \mathrm{D}$ rutile or anatase $\mathrm{MO}_{2}$ phase and the $2 \mathrm{D} s q-\mathrm{MO}_{2}$ phase. For readers' information, all bond lengths and coordination numbers for the $h-\mathrm{MO}, s q-\mathrm{MO}, h-\mathrm{M}_{2} \mathrm{O}_{3}$, and $s q-$ $\mathrm{MO}_{2}$ phases, and their corresponding 3D phases are given in Table $\mathrm{S} 2$ as well.

Table 2 lists the calculated values for $\Delta E$. For all 2D TMOs except three, the calculations resulted in a $\Delta E$ larger than $200 \mathrm{meV} /$ atom. The three 2D TMOs that do meet the $200 \mathrm{meV} /$ atom criterion, however, were found to even have a negative $\Delta E$. These phases are $2 \mathrm{D} s q-\mathrm{TiO}, s q-\mathrm{CrO}$, and $s q-\mathrm{MnO}$, and that they are energetically more favorable than 3D $\epsilon$ - $\mathrm{TiO}, r s-\mathrm{CrO}$, and $r s-\mathrm{MnO}$, indicates that they may be synthesized as stable free-standing slabs. To further understand the underlying reason for the energetic stability of these $s q-\mathrm{MO}$ phases over their corresponding bulk phases, we compared the orbital-resolved density-of-states (DOS) of these 2D TMOs to the DOS of their 3D counterparts. This analysis is included as Supplementary Discussion in the SI.

\section{Thermal stability of the 2D TMO phases}

The thermal stability of the 20 structural and magnetic groundstate configurations was examined by means of AIMD simulation 
at temperatures of 300 and $700 \mathrm{~K}$, and subsequent $0 \mathrm{~K}$ relaxation of AIMD output configurations to see if the structures had remained topologically equivalent. For the structures that did not show bond breaking during AIMD simulation, average bond lengths are listed in Table 3. Graphs showing minimum, average, and maximum bond lengths during 300 and $700-\mathrm{K}$ AIMD simulation can be found in Fig. S1. All 300 and 700-K AIMD output configurations, and all post-AIMD relaxed structures obtained from the $300-\mathrm{K}$ AIMD output configurations, can be found in Fig. S1 as well. Figure 3 shows a selection, and includes for a number of structures perspective views and post-AIMD relaxed structures obtained from $700-\mathrm{K}$ AIMD output configurations, not shown in Fig. S1.

For the sq-MO phases, the AIMD and post-AIMD simulations indicate that planar $s q-\mathrm{TiO}$ and $s q-\mathrm{VO}$ are thermally stable structures. These phases show nice undulation during 300 and $700 \mathrm{~K}$ AIMD simulation, bond lengths increasing with increasing temperature and layers exhibiting out-of-plane displacement of atoms and wave-like vibration of the entire structure, but clearly remain the same: the $0 \mathrm{~K}$ relaxed AIMD output configurations for these phases are very smoothly corrugated 2D square structures that are energetically as favorable as their planar counterparts. Planar $s q-\mathrm{ScO}$ and mono-sided buckled $s q-\mathrm{CrO}$ were found to be thermally unstable: post-AIMD relaxation for these phases resulted in energetically more favorable double-sided oxygen terminated (and for sq-ScO: highly corrugated) structures that are no longer atomically thin (see Fig. $3 c$ ). Metastable $s q-\mathrm{MnO}$ is thermally stable at $300 \mathrm{~K}$ and thermally unstable at $700 \mathrm{~K}$ : at $300 \mathrm{~K}$, this structure remained the same, its AIMD output configuration at $0 \mathrm{~K}$ relaxing into a very smoothly corrugated square structure (see Fig. 3b, d) that is energetically as stable as planar sq-MnO; at $700 \mathrm{~K}, s q-\mathrm{MnO}$ transformed into a hexagonal phase (see Fig. 3a). The 2D square $\mathrm{MnO} \rightarrow 2 \mathrm{D}$ hexagonal $\mathrm{MnO}$ phase transition will be further described below.

The planar $s q-\mathrm{MO}_{2}$ phases are relatively open structures, the number of bonds per atom being two for $\mathrm{O}$ and four for TM atoms

Table 2. Relative stability (eV/atom) of the $2 \mathrm{D}$ TMO phases with respect to the corresponding 3D bulk TMOs listed in Table S2 of the SI

\begin{tabular}{llllc}
\hline $\mathrm{TM}$ & $h-\mathrm{MO}$ & $s q-\mathrm{MO}$ & $h-\mathrm{M}_{2} \mathrm{O}_{3}$ & $s q-\mathrm{MO}_{2}$ \\
\hline $\mathrm{Sc}$ & 0.48 & 0.43 & 0.86 & - \\
$\mathrm{Ti}$ & 0.51 & -1.60 & 0.60 & 0.93 \\
$\mathrm{~V}$ & 0.41 & 0.22 & 0.47 & 0.70 \\
$\mathrm{Cr}$ & 0.27 & -0.02 & 0.59 & 0.57 \\
$\mathrm{Mn}$ & 0.29 & -0.26 & 0.44 & 0.65 \\
\hline
\end{tabular}

Table 3. Average bond lengths in $\AA$ for the structures that showed no bond breaking during AIMD simulation, at 300 and $700 \mathrm{~K}$; showing within parentheses the increase in percent relative to the $0 \mathrm{~K}$ value prior to simulation

\begin{tabular}{|c|c|c|c|c|c|c|c|c|}
\hline & \multicolumn{2}{|l|}{$h-\mathrm{MO}$} & \multicolumn{2}{|l|}{$s q-\mathrm{MO}$} & \multicolumn{2}{|l|}{$h-\mathrm{M}_{2} \mathrm{O}_{3}$} & \multicolumn{2}{|l|}{$s q-\mathrm{MO}_{2}$} \\
\hline Sc & $2.039(+1.1 \%)$ & $2.050(+1.6 \%)$ & $2.138(+1.3 \%)$ & $2.153(+2.0 \%)$ & $1.941(+1.1 \%)$ & $1.958(+2.0 \%)$ & $2.069(+3.3 \%)$ & - \\
\hline $\mathrm{Ti}$ & $1.959(+2.0 \%)$ & $1.970(+2.6 \%)$ & $2.039(+1.0 \%)$ & $2.059(+2.0 \%)$ & $1.847(+1.1 \%)$ & $1.862(+2.0 \%)$ & - & - \\
\hline V & $1.916(+0.4 \%)$ & $1.934(+1.4 \%)$ & $2.002(+0.8 \%)$ & $2.018(+1.6 \%)$ & $1.804(+1.0 \%)$ & $1.818(+1.9 \%)$ & - & - \\
\hline $\mathrm{Cr}$ & $1.921(+2.3 \%)$ & $1.931(+2.7 \%)$ & $2.007(+1.0 \%)$ & $2.019(+1.5 \%)$ & $1.796(+1.3 \%)$ & $1.813(+2.3 \%)$ & - & - \\
\hline
\end{tabular}

Minimum and maximum bond lengths during simulation, and $0-\mathrm{K}$ bond lengths prior to simulation, can be found in Table S3. Bond length graphs are shown in Fig. S1. That bond lengths can be evaluated does not necessarily imply that a structure is thermally stable: a number of structures for which bond lengths are listed in this Table, were found to restructure into energetically more favorable configurations, and as such are considered to be thermally unstable structure could cause a layer to rupture more easily; or to be more resilient to stress, due to increased flexibility. For all $s q-\mathrm{MO}_{2}$ phases except $s q-\mathrm{SCO}_{2}$, it has resulted in the former: during $300-\mathrm{K}$ simulation, $s q-\mathrm{TiO}_{2}, s q-\mathrm{VO}_{2}, s q-\mathrm{CrO}_{2}$, and $s q-\mathrm{MnO}_{2}$, were all found to disintegrate and partly restructure into phases that are no longer atomically thin. $s q-\mathrm{ScO}_{2}$, however, was found to retain structural integrity and square character during $300 \mathrm{~K}$ simulation (bond lengths increasing by $\sim 3 \%$, though); and after $700-\mathrm{K}$ simulation, $s q-\mathrm{ScO}_{2}$ showed only the onset of the disintegration and partial restructuring, which for the other $s q-\mathrm{MO}_{2}$ phases, was already and to a larger extent observed during $300 \mathrm{~K}$ simulation (see Fig. 3e-i). Relaxing at $0 \mathrm{~K}$ again, the $s q-\mathrm{ScO}_{2} 300-\mathrm{K}$ AIMD output configuration was found to restructure into a phase that is no longer square and no longer atomically thin (see Fig. S1), indicating that $s q-\mathrm{ScO}_{2}$ too is thermally unstable.

For the $h-\mathrm{M}_{2} \mathrm{O}_{3}$ phases, the AIMD simulations and post-AIMD relaxations show that planar $h-\mathrm{SC}_{2} \mathrm{O}_{3}$ and $h-\mathrm{V}_{2} \mathrm{O}_{3}$ are thermally stable: the $0-\mathrm{K}$ relaxed output configurations for these phases are very smoothly corrugated atomically thin 2D structures (see Fig. $3 \mathrm{j})$ that are energetically as favorable as their planar counterparts. The buckled $h-\mathrm{Ti}_{2} \mathrm{O}_{3}$ and planar $h-\mathrm{Cr}_{2} \mathrm{O}_{3}$ phases were found to be unstable; during post-AIMD relaxation, the supercells for these phases restructured into phases that are no longer atomically thin (see Fig. 3l). The $h-\mathrm{Mn}_{2} \mathrm{O}_{3}$ phase is thermally stable. Both the 300 and $700-\mathrm{K}$ AIMD output configurations for this phase were found to restructure into arrangements of smoothly tilted hexagons (see Fig. $3 \mathrm{k}, \mathrm{m}$ ) that are only slightly more favorable than planar $h$ $\mathrm{Mn}_{2} \mathrm{O}_{3}$, by $1 \mathrm{meV} /$ atom, which is at the accuracy limit of the calculations. This indicates that the $h-\mathrm{Mn}_{2} \mathrm{O}_{3}$ structure preserves its hexagonal and atomically thin 2D character, i.e., is thermally stable at 300 and $700 \mathrm{~K}$, but will exist as a smoothly corrugated structure rather than as a planar structure.

The $h-\mathrm{MO}$ phases were all found to be thermally unstable. Buckled $h-\mathrm{ScO}$ and $h$-TiO retained structural integrity during AIMD simulation; at $0 \mathrm{~K}$, their AIMD output configurations relaxed, however, into structures that are no longer atomically thin (see Fig. $3 \mathrm{n}$ ) and energetically more favorable than buckled $h-\mathrm{ScO}$ and $h$-TiO. Buckled $h$-VO, and planar $h$-CrO and $h-\mathrm{MnO}$, also retained structural integrity during AIMD simulation and subsequently relaxed into energetically more favorable configurations; the AIMD output configurations for these three phases, however, were found to have restructured upon post-AIMD relaxation into atomically thin supercells of hexagonal " $t-M O$ " phases displaying trigonal rotation symmetry (see Fig. 3o-q) that are thermally stable. (These three $t-\mathrm{MO}$ phases will be further discussed below.)

In summary (see also Table 1 ), of the $20 h-\mathrm{MO}, s q-\mathrm{MO}, h-\mathrm{M}_{2} \mathrm{O}_{3}$, and $s q-\mathrm{MO}_{2}$ structural and magnetic ground-state configurations, 5 were found to be thermally stable, 1 to be thermally stable at $300 \mathrm{~K}$ and thermally unstable at $700 \mathrm{~K}$, and 14 to be unstable. The unstable 
(a) $s q-\mathrm{MnO}$ at $700 \mathrm{~K}$
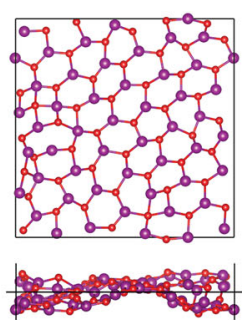

(e) $s q-\mathrm{ScO}_{2}$ at $300 \mathrm{~K}$

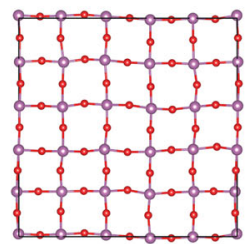

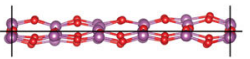

(b) $s q-\mathrm{MnO}$ at $0 \mathrm{~K}$ after $300 \mathrm{~K}$
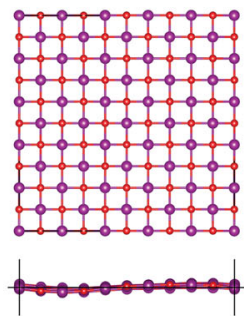

(f) $s q-\mathrm{ScO}_{2}$ at $700 \mathrm{~K}$

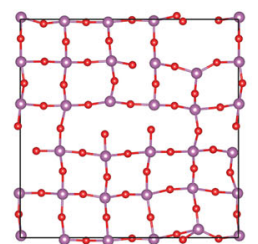

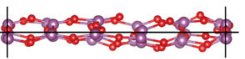

(c) $s q-\mathrm{CrO}$

at $0 \mathrm{~K}$ after $700 \mathrm{~K}$

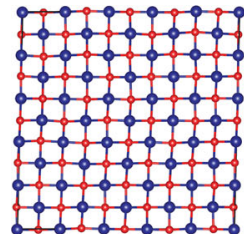

15 .

(g) $s q-\mathrm{TiO}_{2}$ at $300 \mathrm{~K}$
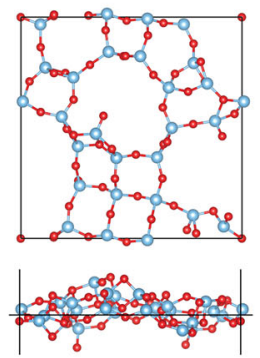

(d) $s q-\mathrm{MnO}$

at $0 \mathrm{~K}$ after $300 \mathrm{~K}$

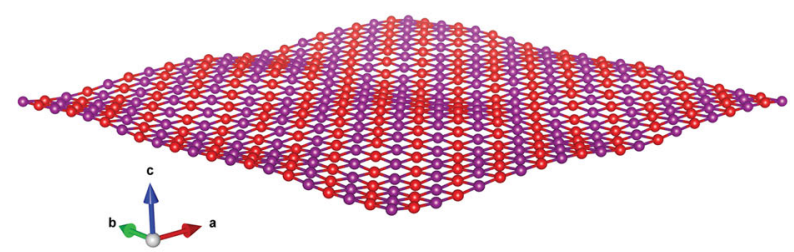

(j) $h-\mathrm{V}_{2} \mathrm{O}_{3}$

at $0 \mathrm{~K}$ after $300 \mathrm{~K}$ (k) $h-\mathrm{Mn}_{2} \mathrm{O}_{3}$ at $0 \mathrm{~K}$ after $300 \mathrm{~K}$ (l) $h-\mathrm{Ti}_{2} \mathrm{O}_{3}$ at $0 \mathrm{~K}$ after $300 \mathrm{~K}$

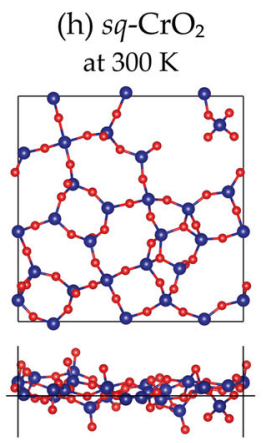

(i) $s q-\mathrm{ScO}_{2}$

bond lengths $(\AA)$ at $300 \mathrm{~K}$

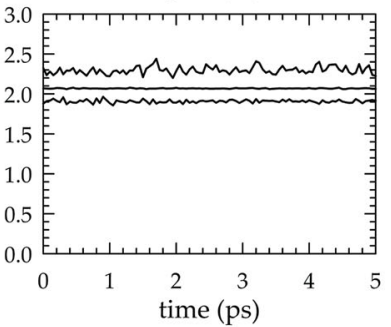

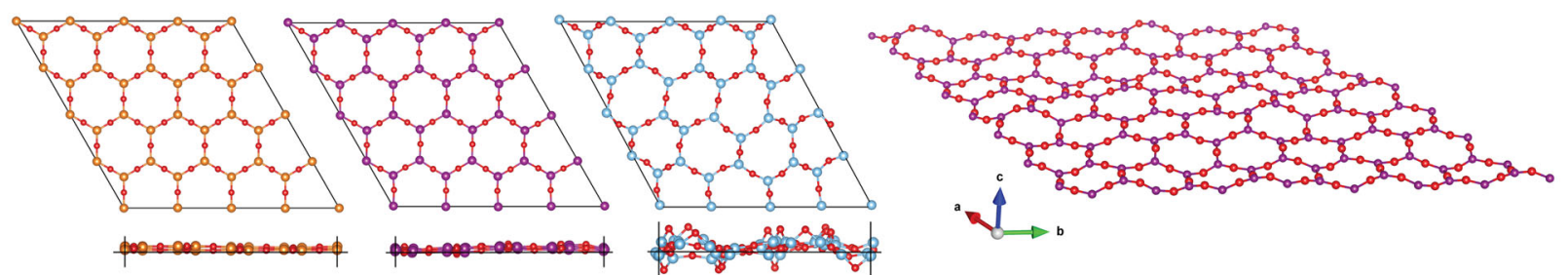

(n) $h-\mathrm{TiO}$

at $0 \mathrm{~K}$ after $300 \mathrm{~K}$ (o) $h$-VO

at $0 \mathrm{~K}$ after $300 \mathrm{~K}$ (p) $h-\mathrm{CrO}$ at $0 \mathrm{~K}$ after $300 \mathrm{~K}$ (q) $h-\mathrm{MnO}$ at $0 \mathrm{~K}$ after $300 \mathrm{~K}$

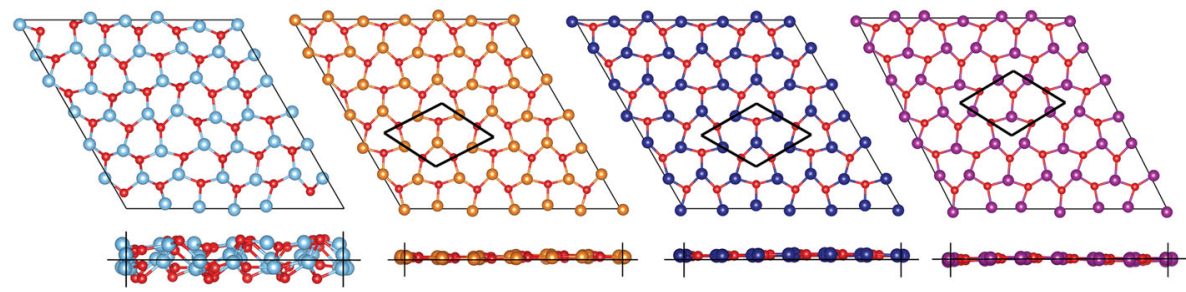

(m) $h-\mathrm{Mn}_{2} \mathrm{O}_{3}$ at $0 \mathrm{~K}$ after $700 \mathrm{~K}$ 
$0.20 \mathrm{ps}$

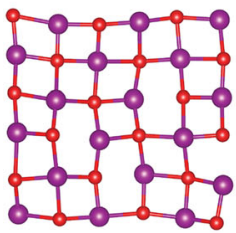

$0.30 \mathrm{ps}$

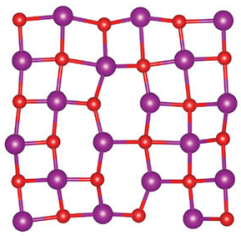

$0.40 \mathrm{ps}$

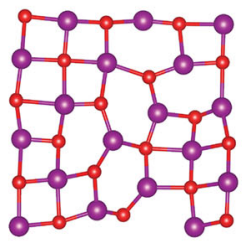

$0.50 \mathrm{ps}$

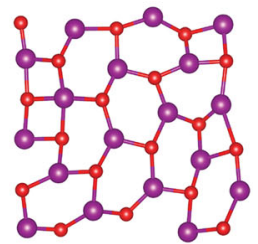

Fig. 4 Snapshots of the $700 \mathrm{~K}$ equilibration of $s q-\mathrm{MnO}$, illustrating the mechanism of transformation of $2 \mathrm{D}$ square $s q-\mathrm{MnO}$ into $2 \mathrm{D}$ hexagonal $t$-MnO. The transformation is also shown in Supplementary Video 1

(a)

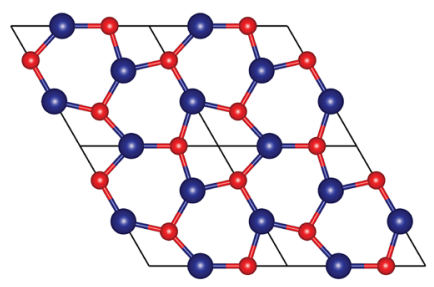

$t-\mathrm{MO}(\mathrm{p} 31 \mathrm{~m})$

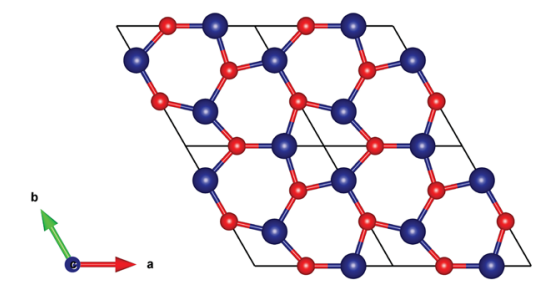

AFM1

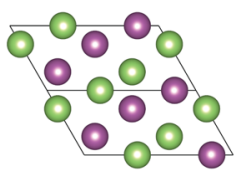

AFM2

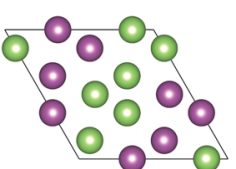

Fig. 5 Input geometry and considered anti-ferromagnetic orderings for the two atomic arrangements of the $t$-MO phase. Dark blue and red spheres denote TM and $\mathrm{O}$ atoms, respectively. Light green and purple spheres denote spin-up and spin-down TM atoms, respectively. Boundaries of simulation and unit cells are indicated with black solid lines

$400 \mathrm{~K}$ for the transformation temperature was obtained: continuing the $300-\mathrm{K}$ simulation for another $50 \mathrm{ps}$, still no transformation of sq-MnO was observed; while during a $10 \mathrm{ps}$ simulation at $400 \mathrm{~K}$ after initial 2 -ps equilibration, transformation into hexagonal $\mathrm{MnO}$ did take place.

Energetic stability of the $t$-MO phases

The $h-\mathrm{VO}, h-\mathrm{CrO}$, and $h-\mathrm{MnO}$ AIMD output configurations were found to restructure, upon post-AIMD relaxation, into atomically thin supercells of hexagonal " $t-\mathrm{MO}^{\prime}$ " phases. As can be seen from Fig. S2, the post-AIMD relaxed $h-\mathrm{MnO}$ supercell has an antiferromagnetic ordering corresponding to the AFM ordering found for $h$-MnO. The post-AIMD-relaxed $h$-VO output configuration was found to be FM ordered, just like $h$-VO, with the exception of one small domain. The post-AIMD relaxation output configuration obtained for anti-ferromagnetic $h$-CrO was found to have antiferromagnetic ordering of a type, however, that was not considered for the $h$-MOs.

To check the structural and magnetic ground state of the three $t$-MOs, total-energy calculations were performed, considering NM, FM, AFM1, and AFM2 magnetic ordering, planar and buckled input configurations, and two types of atomic arrangements (labeled "(a)" and "(b)" as in Fig. 5) for each of the $t$-MOs, bringing the total number of input configurations considered for the $t$-MO phases to 48 (and the total number of input configurations considered in our study to 198). Unit cell geometries and considered AFM orderings are illustrated in Fig. 5. Note that the $t$-MO phase is a $\sqrt{3} \times \sqrt{3}$ reconstruction of the $h-\mathrm{MO}$ phase, lowering the $\mathrm{p} 3 \mathrm{~m} 1$ symmetry for perfect $h-\mathrm{MO}$ to $\mathrm{p} 31 \mathrm{~m}$ symmetry for perfect $t-\mathrm{MO}$.

The results of the total-energy calculations can be found in Table S1. Structural parameters for the structural and magnetic ground-state configurations are listed in Table 4. The ground-state
Table 4. PBE-calculated lattice parameters and atom coordinates for the $t$-MO phases

\begin{tabular}{llll}
\hline & $t$-VO & $t$-CrO & $t$-MnO \\
\hline$a(\AA)$ & 5.423 & 5.435 & 5.710 \\
$b(\AA)$ & - & - & 5.447 \\
$\gamma\left({ }^{\circ}\right)$ & 119.5 & 119.8 & 118.5 \\
$(x, y) \mathrm{M} 1$ & $(0.388,0.004 ;-0.02)$ & $(0.386,0.001)$ & $(0.716,0.999)$ \\
$(x, y) \mathrm{M} 2$ & $(0.004,0.388 ;-0.02)$ & $(0.001,0.386)$ & $(0.000,0.730)$ \\
$(x, y) \mathrm{M} 3$ & $(0.608,0.608 ; 0.02)$ & $(0.614,0.614)$ & $(0.284,0.282)$ \\
$(x, y) \mathrm{O} 1$ & $(0.724,0.999 ;-0.14)$ & $(0.718,0.997)$ & $(0.369,0.989)$ \\
$(x, y) \mathrm{O} 2$ & $(0.999,0.724 ;-0.14)$ & $(0.997,0.718)$ & $(0.000,0.379)$ \\
$(x, y) \mathrm{O} 3$ & $(0.276,0.276 ; 0.44)$ & $(0.283,0.283)$ & $(0.631,0.620)$ \\
\hline
\end{tabular}

To make comparison between the $t$-MO phases easier and to emphasize their near-trigonal rotation symmetry, the structure parameters are of primitive unit cells, given in $P 1$ and $\mathrm{p} 1$ symmetry with the atoms at Wyckoff 1 a positions. Note that because of its monolayer character, the $z$ coordinates for $t$-VO are absolute coordinates in $\AA$, whereas $x$ and $y$ are relative coordinates

configurations for $t$ - $\mathrm{VO}$ and $t$ - $\mathrm{CrO}$ were found to be atomic arrangements as depicted in Fig. 5a; the ground-state configuration for $t-\mathrm{MnO}$ was found to be an atomic arrangement as depicted in Fig. $5 b$. $t$ - $\mathrm{CrO}$ and $t-\mathrm{MnO}$ are planar structures. The lowest-energy configuration for $t$ - $\mathrm{VO}$, however, was found to be a buckled phase, of a type where only one-third of the oxygen atoms (i.e., the oxygen atoms at the $x=y$ mirror plane) are located at the buckling distance of $0.44 \AA$ (see Table 4; Fig. S2). The $t-\mathrm{MnO}$ phase was found to have an AFM1 ordering corresponding to its 
$h$-MO precursor's magnetic ground state. $t$ - $\mathrm{CrO}$ was found to have an anti-ferromagnetic ordering of a type (AFM2) that was not considered for the $h$-MOs ( $h$-CrO has an anti-ferromagnetic ordering as well, of the AFM1 type, however). $t$-VO is also AFM2 ordered; in contrast to its $h$-VO precursor, which has FM ordering.

Comparing the formation energies of the $t-M O$ phases and their corresponding $h-\mathrm{MO}$ and $s q-\mathrm{MO}$ phases, it can be observed from Table 1 and Fig. 2 , that just like their corresponding $h$-MO phases, $t$ - $\mathrm{VO}$ and $t$-CrO are energetically less favorable, and $t-\mathrm{MnO}$ is energetically more favorable, than the corresponding sq-MO phase. $t-\mathrm{VO}, t-\mathrm{CrO}$, and $t-\mathrm{MnO}$, are all energetically more favorable than their corresponding $h-\mathrm{MO}$ phases, by 74,155 , and $71 \mathrm{meV} /$ atom, respectively. The (latter) energetic stability of the $t-\mathrm{MO}$ phases over the $h$-MO phases may be due to the PJTE (for a brief discussion of the PJTE: see the section on the energetic stability of the $h-\mathrm{MO}, s q-\mathrm{MO}, h-\mathrm{M}_{2} \mathrm{O}_{3}$, and $s q-\mathrm{MO}_{2}$ phases above).

\section{Discussion of stability and methodology}

The AIMD simulations and post-AIMD relaxations indicate that nine of the 2D TMO structural and magnetic ground-state configurations identified in this study are thermally stable (see Table 1). These phases not only retained structural integrity, i.e., did not break at elevated temperatures, but also preserved their atomically thin, and hexagonal or square, 2D character. Our findings suggest that these structures may all be synthesized as free-standing atomically thin nanosheets showing smooth corrugation upon realization. The thermally unstable $2 \mathrm{D}$ TMO phases can most likely not exist as free-standing slabs. These phases, however, may be stabilized using an appropriate support: substrate stabilization of 2D materials can be as large as $8.84 \mathrm{~kJ} /$ mol-atom (91.6 meV/atom); ${ }^{59}$ and substrates can be chosen to interact with the synthesized 2D TMO thin film via only a weak van der Waals force, which would stabilize the film without much interference with its electronic structure. ${ }^{60}$ The thermally stable phases were found to include 2D TMOs that do not meet the 200 meV criterion (see the section on the energetic stability of 2D TMO phases with respect to bulk); and $s q-\mathrm{CrO}$, which does meet the $200 \mathrm{meV}$ criterion, was found to be thermally unstable. This merely indicates, however, that comparing 2D TMOs with corresponding bulk phases is not that straightforward, and that for a number of 2D TMO phases perhaps the corresponding bulk phase has not been chosen properly.

The AIMD as performed, imposing periodic boundary conditions on the structures, tends to overestimate structural stability: lateral periodicity mitigates out-of-plane movements of the atoms and artificially enhances structural integrity, and may result in structural integrity for structures that would otherwise curl up or even disintegrate. Furthermore, the results presented here are the outcome of $7 \mathrm{ps}$ simulations; prolonged simulations might yield different results with respect to the stability of the structures. We are confident about our approach consisting of AIMD simulation and subsequent relaxation of AIMD output configurations, though, because of the proper prediction of $h-\mathrm{V}_{2} \mathrm{O}_{3}$ : of all the structural and magnetic ground-state 2D TMO configurations identified in this study, only $h-\mathrm{V}_{2} \mathrm{O}_{3}$ has been experimentally realized so far, ${ }^{15,16,23}$ and our simulations indeed resulted in thermal stability for this phase. That $h-\mathrm{V}_{2} \mathrm{O}_{3}$ has been realized in a buckled form while our simulations indicate that the planar configuration is the energetically more favorable configuration for this phase, does not detract from this: our calculations suggest that $h-\mathrm{V}_{2} \mathrm{O}_{3}$ may be realized as free-standing smoothly corrugated planar nanosheets; the reported buckling of $h-\mathrm{V}_{2} \mathrm{O}_{3}$ may be due to its realization on a $\operatorname{Pd}(111)$ support.

Our method of AIMD simulation and subsequent relaxation of AIMD output configurations yields more reliable stability results than approaches that depend on AIMD simulation alone: Of all the 2D TMO phases identified in this study, other than $h-\mathrm{V}_{2} \mathrm{O}_{3}$, only $h$ -
MnO has been found reported on before, in a study that based on AIMD simulation only and finding no structure destruction concluded $h-\mathrm{MnO}$ to be thermally stable up to temperatures of at least $500 \mathrm{~K}^{28}$ Our method, however, indicates that $h-\mathrm{MnO}$ is thermally unstable at $300 \mathrm{~K}$, already, and that any future experimental realization of an atomically thin $2 \mathrm{D}$ hexagonal $\mathrm{MnO}$ will more likely be of $\mathrm{MnO}$ in the $t-\mathrm{MO}$ configuration. Besides $h-\mathrm{MnO}, 15$ more of the $h-\mathrm{MO}, s q-\mathrm{MO}, h-\mathrm{M}_{2} \mathrm{O}_{3}$, and $s q-\mathrm{MO}_{2}$ groundstate configurations were found to show no structure destruction during $300 \mathrm{~K}$ AIMD simulation; only six of these, however, were also found to be thermally stable at $300 \mathrm{~K}$. The discovery of the thermally stable $t-\mathrm{MO}$ phases in addition shows that post-AIMD relaxation of AIMD output configurations may also reveal new phases that otherwise would have remained undisclosed.

\section{Electronic and magnetic properties of the 2D TMOs}

To further investigate the magnetic properties of the 2D TMOs and to disclose their electronic structure, we calculated the electronic band structures and determined the bandgaps and atomic magnetic moments and Bader charges of the magnetic ground-state configurations, using the PBE and HSE06 functionals. A summary of the HSE-obtained results can be found in Table 1 and Fig. 6. Table S4 and Fig. S3 include the PBE results and orbitalresolved band structures.

From the comparison made in Table S4 and Fig. S3 between PBE and HSE-calculated electronic and magnetic properties, it can be seen that, in terms of an enlarged bandgap, HSE gives a better description of the strongly correlated $d$ electrons than PBE. In particular, HSE opened bandgaps for sq- $\mathrm{MnO}, h-\mathrm{Cr}_{2} \mathrm{O}_{3}, s q-\mathrm{TiO}_{2}, s q-$ $\mathrm{VO}_{2}$, and $s q-\mathrm{MnO}_{2}$, making these phases no longer metals as predicted by $\mathrm{PBE}$, but semiconductors, semimetals, and halfmetals instead. The qualitative discrepancy between PBE and HSE occurs only when describing the electronic ground state of the 2D TMOs. For the magnetic ground state, the two approaches (i.e., PBE and HSE band-structure calculation of an identical PBE-relaxed atomic configuration) always give the same results. Regarding the Bader analysis: HSE always gives values for the atomic partial charges that are slightly larger (by $0.1-0.3 e$ ) than PBE-calculated values. Similar to the electronic charges, the magnetic moments on the atoms (that were obtained by evaluating spin-up and spindown electron densities inside Bader volumes) were also found to be slightly higher (by $0.2-0.5 \mu_{B}$ ) when using HSE in comparison with PBE-calculated values. Our results therefore suggest that for obtaining a qualitative description of the magnetism and charge distribution of the 2D TMOs, DFT calculations suffice. Gaining a qualitatively correct picture of the electronic structure, however, requires more advanced methods such as using the hybrid HSE06 functional that incorporates a contribution of HF exchange. The effect of this HF correction on a particular TMO phases depends on the elements, their valence states, the levels of the atomic orbitals, as well as on the crystal geometry. The general trend, however, is that the HF correction lowers the energy levels of occupied states and lifts the levels of unoccupied states, and has a strong impact on the energy levels of the itinerant $d$ electrons. The partial atomic charges and the magnetic moments on the atoms are determined from (total, and spin-up and spin-down) electron densities inside Bader atomic volumes. As the main effect of the $\mathrm{HF}$ correction is on the eigenvalues of the states rather than on the electron-density distribution, the partial charges and the local magnetic moments obtained from HSE calculations are quite similar to those obtained by PBE calculations (and result in quite similar structural properties as well), while the impact on the electronic band structure is in general very significant.

Using the hybrid HSE06 functional, we found a rich variety of electronic properties among the 2D TMO phases. As can be seen from Table 1 and Fig. 6, of the 23 ground-state configurations, fifteen structures are predicted to be semiconductors and three to 

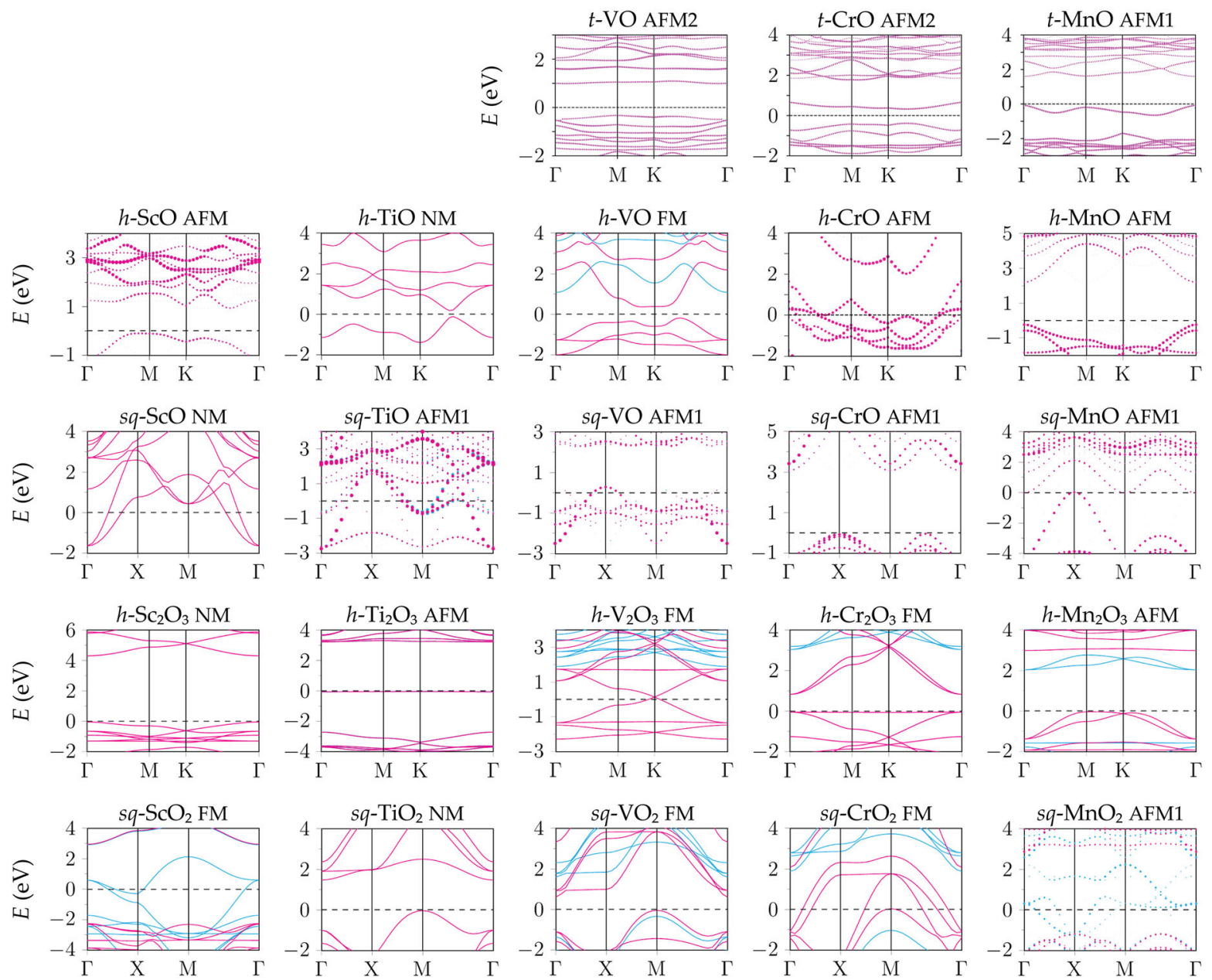

Fig. 6 HSE-calculated electronic band structures of the 2D TMO magnetic ground-state configurations. The Fermi level is set to zero and indicated with a dashed line. For spin-polarized configurations, spin-down bands are shown in cyan when spin-up and spin-down bands do not overlap

be metals with various magnetic orderings; sq- $\mathrm{MnO}$ was found to be an anti-ferromagnetic semimetal; and $h-\mathrm{V}_{2} \mathrm{O}_{3}, \mathrm{sq}-\mathrm{ScO}_{2}, \mathrm{sq}-\mathrm{CrO}_{2}$, and $s q-\mathrm{MnO}_{2}$, are half-metals that are either ferromagnets $\left(h-\mathrm{V}_{2} \mathrm{O}_{3}\right.$, $s q-\mathrm{ScO}_{2}$, and $\left.s q-\mathrm{CrO}_{2}\right)$ or antiferromagnets $\left(s q-\mathrm{MnO}_{2}\right)$. The magnetic moments on the atoms were evaluated from the spinup and spin-down electron densities within Bader atomic volumes, and vary up to values of $4.6 \mu_{B}$ for the transition metal atoms. Besides the four half-metals, four semiconductors were also found to have non-overlapping spin-up and spin-down bands and different bandgaps for spin-up and spin-down electrons; these semiconductors are: ferromagnetic $h$-VO, $h-\mathrm{Cr}_{2} \mathrm{O}_{3}$, and $s q$ $\mathrm{VO}_{2}$, and ferrimagnetic $h-\mathrm{Mn}_{2} \mathrm{O}_{3}$. For the ferromagnetic half-metal $h-\mathrm{V}_{2} \mathrm{O}_{3}$, and for the non-magnetic semiconductor $h-\mathrm{TiO}$, Diraccone-like shaped dispersion curves were found. All these remarkable properties indicate that the 2D TMOs are bright candidates for electronic, magnetic, and spintronic applications. Exceptionally remarkable in this regard is $h-\mathrm{V}_{2} \mathrm{O}_{3}$, that was found to be thermally stable up to temperatures of $700 \mathrm{~K}$, and combines magnetism and a massless behavior of spin-up electrons along with a wide bandgap of $6.2 \mathrm{eV}$ for spin-down electrons.

To understand the half-metallic property of $h-\mathrm{V}_{2} \mathrm{O}_{3}, s q-\mathrm{ScO}_{2}, s q-$ $\mathrm{CrO}_{2}$, and $s q-\mathrm{MnO}_{2}$ in more detail, we performed a spinpolarization analysis of their density-of-states (DOS) at the Fermi level. The spin polarization $P$ is defined as

$P=\frac{D \uparrow\left(E_{F}\right)-D \downarrow\left(E_{F}\right)}{D \uparrow\left(E_{F}\right)+D \downarrow\left(E_{F}\right)}$, where $D\left(E_{F}\right)$ is the magnitude of the DOS at the Fermi level for spin-up $(\uparrow)$ and spin-down $(\downarrow)$ states. The spin polarization serves as a criterion to determine the extent of spin polarization, hence the degree of half-metallicity, of a material. ${ }^{61-63} P= \pm 1$ indicates that a system is half-metallic, whereas $P=0$ shows that a system has an equal amount of spin-up and spin-down states at the Fermi level. DOS analysis indeed showed that for $h-\mathrm{V}_{2} \mathrm{O}_{3}, s q-\mathrm{ScO}_{2}, s q-$ $\mathrm{CrO}_{2}$, and $s q-\mathrm{MnO}_{2}$, the spin polarization is equal to the ideal value of 1.

We have presented the results from our systematic firstprinciples study of atomically thin 2D TMOs. For the five early $3 d$ transition metals $\mathrm{Sc}, \mathrm{Ti}, \mathrm{V}, \mathrm{Cr}$, and $\mathrm{Mn}$, four different 2D TMO phases were designed, namely $h-\mathrm{MO}, s q-\mathrm{MO}, h-\mathrm{M}_{2} \mathrm{O}_{3}$, and $s q-\mathrm{MO}_{2}$, and we investigated their structural and magnetic ground-state configurations, considering various magnetic orderings and planar and mono-sided buckled configurations. The 20 structural and magnetic ground-state configurations for these phases were all found to have negative formation energies with respect to the elemental transition metal bulk phases and the paramagnetic oxygen molecule. The concave-hull plot of these formation energies indicates that all the 2D TMOs are energetically stable at their respective stoichiometries.

AIMD simulation at temperatures of 300 and $700 \mathrm{~K}$, and subsequent $0 \mathrm{~K}$ relaxation of AIMD output configurations, were carried out to examine the thermal stability of the 2D TMO phases. Of the $20 h-\mathrm{MO}, s q-\mathrm{MO}, h-\mathrm{M}_{2} \mathrm{O}_{3}$, and $s q-\mathrm{MO}_{2}$ ground-state configurations, 5 were found to be thermally stable; metastable 
sq-MnO was found to be thermally stable at $300 \mathrm{~K}$ and thermally unstable at $700 \mathrm{~K}, 2 \mathrm{D}$ square sq-MnO transforming into a 2D hexagonal $\mathrm{MnO}$ phase at temperatures of $400 \mathrm{~K}$ and higher; and 14 configurations were found to be thermally unstable. The unstable configurations include all $s q-\mathrm{MO}_{2}$ phases, and all $h-\mathrm{MO}$ phases. The post-AIMD relaxations revealed a $2 \mathrm{D}$ hexagonal $t-\mathrm{MO}$ phase that is a $\sqrt{3} \times \sqrt{3}$ reconstruction of the $h$-MO phase; however, that is thermally stable for $\mathrm{V}, \mathrm{Cr}$, and $\mathrm{Mn}$. This brings the total number of 2D TMOs identified in this study that may be realized as free-standing nanosheets to nine. These phases are: $s q$ $\mathrm{TiO}, s q-\mathrm{VO}$, and $s q-\mathrm{MnO} ; h-\mathrm{Sc}_{2} \mathrm{O}_{3}, h-\mathrm{V}_{2} \mathrm{O}_{3}$, and $h-\mathrm{Mn}_{2} \mathrm{O}_{3}$; and $t$-VO, $t$ - $\mathrm{CrO}$, and $t-\mathrm{MnO}$; of all these, only $h-\mathrm{V}_{2} \mathrm{O}_{3}$ has been experimentally realized so far. Our method of AIMD simulation and subsequent relaxation of AIMD output configurations yields more reliable results than approaches that depend on AIMD simulation alone: Of all the 2D TMO phases identified in this study, other than $h-\mathrm{V}_{2} \mathrm{O}_{3}$, only $h-\mathrm{MnO}$ was found reported on before, in a study that, based on AIMD simulation only, predicts $h-\mathrm{MnO}$ to be thermally stable. Our method, however, indicates that $h-\mathrm{MnO}$ is thermally unstable, and that any future experimental realization of atomically thin 2D hexagonal $\mathrm{MnO}$ will more likely be of $\mathrm{MnO}$ in the $t$ MO phase. Our findings furthermore suggest that all thermally stable phases will show smooth corrugation upon realization. The thermally unstable 2D TMO phases can most likely not exist as free-standing slabs but may very well be stabilized when using an appropriate support.

A rich variety of electronic and magnetic properties was discovered for the 2D TMOs identified in this study, using the HSE06 hybrid functional. In particular, $s q-\mathrm{MnO}$ is predicted to be a semimetal with anti-ferromagnetic ordering; $h-\mathrm{V}_{2} \mathrm{O}_{3}, s q-\mathrm{ScO}_{2}$, and $s q-\mathrm{CrO}_{2}$ were found to be ferromagnetic half-metals, while $s q$ $\mathrm{MnO}_{2}$ is an anti-ferromagnetic half-metal; and Dirac-cone-like bands were found for the non-magnetic semiconductor $h$-TiO and the ferromagnetic half-metal $h-\mathrm{V}_{2} \mathrm{O}_{3}$. The magnetic moments on the transition metal atoms vary up to values of $4.6 \mu_{B}$. All these remarkable properties indicate that the 2D TMOs are bright candidates for electronic, magnetic, and spintronic applications. Exceptionally remarkable in this regard is planar $h-\mathrm{V}_{2} \mathrm{O}_{3}$, that combines magnetism and a massless behavior of spin-up electrons along with a wide bandgap of $6.2 \mathrm{eV}$ for spin-down electrons, and was found to be thermally stable up to temperatures of $700 \mathrm{~K}$. The hybrid HSE06 functional gives a scenario of the electronic properties for the 2D TMOs that significantly differs from the one obtained using DFT: in general, HSE successfully opened up a bandgap for materials which were predicted to be metals by traditional DFT. This shows the necessity of using more advanced electronic structure methods to treat these strongly correlated systems correctly.

The current study assessed the stability of the 2D TMO phases and highlighted their remarkable electronic and magnetic properties, presenting an opportunity for experimentalists of targeted synthesis of these novel 2D TMO materials, which hold great potential for electronic and spintronic applications. For future work, a thorough study of the growth process and substrate effects is crucial to the successful realization of these 2D nanomaterials. Furthermore, a comprehensive study of point defects such as monovacancies, interstitials, antisites, and dopants, and the mutual interaction between these different kinds of defects, will open up the possibility of successful defect engineering in these $2 \mathrm{D}$ TMOs.

\section{METHODS}

Total-energy calculations

The DFT calculations were performed using the first principles' Vienna Ab initio Simulation Package $e^{31-33}$ (VASP). Within the projector-augmented wave (PAW) framework, ${ }^{34,35}$ the exchange and correlation energy terms were described using the PBE generalized gradient approximation (GGA) functional. ${ }^{36,37}$ The cutoff energy of the wave functions was set to $700 \mathrm{eV}$, and the cutoff energy of the augmentation functions to $850 \mathrm{eV}$. All calculations were carried out using conventional unit cells or $2 \times$ 2 supercells, dependent on the input configurations' magnetic ordering; except for $h$-MO, which requires a $3 \times 3$ supercell to accommodate the FiM ordering. Figure 1 illustrates the considered anti-ferromagnetic orderings for the four 2D TMO input geometries and their corresponding simulation cell sizes. Different starting values for the magnitudes of the magnetic moments on the TM atoms were considered as well, with input values of $0.0 \mu_{B}$ for non-magnetic calculations, and input values of 1.0, 2.0, 3.0, 4.0, and $5.0 \mu_{B}$ for magnetic calculations. To avoid spurious interactions between layers, a vacuum slab that is larger than $20 \AA$ was included in all simulation cells. The electronic wave functions were sampled on grids in inverse proportion to simulation cell size, using a $16 \times 16 \times 1 \Gamma$-centered $k$ mesh for the conventional unit cells. Cutoff energies, $k$-mesh, and height of the vacuum slab were all tested to ensure energy convergence within $1 \mathrm{meV} /$ atom. The structures were structurally and magnetically optimized, keeping the cell volume fixed to avoid collapse of the vacuum slab separating the model layers, and using energy and atomic force convergence criteria of $10^{-6} \mathrm{eV}$ and $10^{-2} \mathrm{eV} / \AA \mathrm{A}$, respectively. The calculations are valid for a temperature of $0 \mathrm{~K}$ and a pressure of $0 \mathrm{GPa}$. Zero-point vibration contributions have been neglected. Non-collinear magnetism and spin-orbit coupling have not been considered.

\section{Band-structure analysis}

The electronic structure of the PBE-relaxed configurations was evaluated using the hybrid HSE06 functional. ${ }^{26}$ The fraction of HF exchange for these calculations was set to 0.25 , resulting in a $\frac{1}{4}: \frac{3}{4}$ hybridization of the $\mathrm{HF}$ exchange energy and PBE exchange energy, and the cutoff energy of the wave functions and the augmentation functions to 500 and $600 \mathrm{eV}$, respectively. Electronic band structures obtained from supercells were unfolded using the method by Tomić et al. ${ }^{64}$

\section{AIMD simulations}

The AIMD simulations were performed using 72 -atom $6 \times 6$ supercells for the $h$-MO phases, 100 -atom $5 \times 5$ supercells for the $s q$-MO phases, 80 -atom $4 \times 4$ supercells for the $h-\mathrm{M}_{2} \mathrm{O}_{3}$ phases, and 72 -atom $5 \times 5$ supercells for each of the $s q-\mathrm{MO}_{2}$ phases, except $s q-\mathrm{MnO}_{2}$, which was simulated using a 108 -atom $6 \times 6$ supercell to accommodate its magnetic ordering. For each of the structures, with a time step of $1 \mathrm{fs}$, a $2 \mathrm{ps}$ initial equilibration and a 5 ps subsequent canonical (NVT) ensemble simulation using a Nosé thermostat ${ }^{49-51}$ was carried out, at simulation temperatures of 300 and $700 \mathrm{~K}$. The AIMD calculations were performed including the $\Gamma$-point only and using a cutoff energy of $400 \mathrm{eV}$ for the wave functions and a cutoff energy of $560 \mathrm{eV}$ for the augmentation functions. After the AIMD simulations, AIMD supercell output configurations were relaxed at high accuracy at $0 \mathrm{~K}$ again, to see if the structures had remained topologically equivalent.

\section{DATA AVAILABILITY}

Details on the crystal structures and calculation settings are provided here and the Supplementary Information. The calculation data will be stored in the Data Repository of Utrecht University, and are available from the authors upon request.

\section{ACKNOWLEDGEMENTS}

This project is financially supported by the Dutch science foundation NWO via a VIDI grant (grant no. 723.012.006) and by the European Research Council through an ERC Consolidator Grant (grant no. 683076). This work was carried out on the Dutch national e-infrastructure with the support of SURF Cooperative and NWO Rekentijd (grant no. 16905). Figures of atomic structural models were produced using VESTA. ${ }^{65}$ W.-F.L. gratefully acknowledges J.-P. Chou and S. S. Gupta for valuable discussions. The authors furthermore appreciate the careful reading and helpful comments on the paper from A. van Blaaderen.

\section{AUTHOR CONTRIBUTIONS}

H.v.G. performed the AIMD and post-AIMD calculations, including the total-energy calculations and band-structure analysis for the $t$-MO phases. W.F.L., M.A.v.H., and H.v. $\mathrm{G}$. performed the total-energy calculations and band-structure analysis for the $h$-MO, $s q-\mathrm{MO}, h-\mathrm{M}_{2} \mathrm{O}_{3}$, and $s q-\mathrm{MO}_{2}$ phases. H.v.G. wrote the paper, incorporating an early 
version of the paper by W.F.L. All authors contributed to discussions and commented on the paper.

\section{ADDITIONAL INFORMATION}

Supplementary information accompanies the paper on the npj $2 D$ Materials and Applications website (https://doi.org/10.1038/s41699-019-0100-z).

Competing interests: The authors declare no competing interests.

Publisher's note: Springer Nature remains neutral with regard to jurisdictional claims in published maps and institutional affiliations.

\section{REFERENCES}

1. Novoselov, S. K. et al. Electric field effect in atomically thin carbon films. Science 306, 666-669 (2004)

2. Butler, S. Z. et al. Progress, challenges, and opportunities in two-dimensional materials beyond graphene. ACS Nano 7, 2898-2926 (2013).

3. Bhimanapati, G. R. et al. Recent advances in two-dimensional materials beyond graphene. ACS Nano 9, 11509-11539 (2015).

4. Mott, N. F. Metal-insulator transition. Rev. Mod. Phys. 40, 677-683 (1968).

5. Imada, M., Fujimori, A. \& Tokura, Y. Metal-insulator transitions. Rev. Mod. Phys. 70 , 1039-1263 (1998)

6. Xu, S., Shen, X., Hallman, K. A., Haglund, R. F. Jr. \& Pantelides, S. T. Unified bandtheoretic description of structural, electronic, and magnetic properties of vanadium dioxide phases. Phys. Rev. B 95, 125105 (2017).

7. Park, J. H. et al. Measurement of a solid-state triple point at the metal-insulator transition in $\mathrm{VO}_{2}$. Nature 500, 431-434 (2013).

8. Wegkamp, D. et al. Instantaneous band gap collapse in photoexcited monoclinic $\mathrm{VO}_{2}$ due to photocarrier doping. Phys. Rev. Lett. 113, 216401 (2014).

9. Bahlawane, N. \& Lenoble, D. Vanadium oxide compounds: structure, properties, and growth from the gas phase. Chem. Vap. Depos. 20, 299-311 (2014)

10. Schwingenschlögl, U. \& Eyert, V. The vanadium Magnéli phases $\mathrm{V}_{n} \mathrm{O}_{2 n-1}$. Ann Phys. (Leipz.) 13, 475-510 (2004).

11. Kitchaev, D. A. et al. Energetics of $\mathrm{MnO}_{2}$ polymorphs in density functional theory. Phys. Rev. B 93, 045132 (2016).

12. Li, Y.-F., Zhu, S.-C. \& Liu, Z.-P. Reaction network of layer-to-tunnel transition of $\mathrm{MnO}_{2}$. J. Am. Chem. Soc. 138, 5371-5379 (2016).

13. Chang, H. L. M., You, H., Guo, J. \& Lain, D. J. Epitaxial $\mathrm{TiO}_{2}$ and $\mathrm{VO}_{2}$ films prepared by MOCVD. Appl. Surf. Sci. 48/49, 12-18 (1991).

14. Makarevich, A. M. et al. Chemical synthesis of high quality epitaxial vanadium dioxide films with sharp electrical and optical switch properties. J. Mater. Chem. C. 3, 9197-9205 (2015).

15. Surnev, S. et al. Growth and structure of ultrathin vanadium oxide layers on Pd (111). Phys. Rev. B 61, 13945-13954 (2000).

16. Surnev, S., Kresse, G., Ramsey, M. G. \& Netzer, F. P. Novel interface-mediated metastable oxide phases: vanadium oxides on Pd(111). Phys. Rev. Lett. 87, 086102 (2001).

17. Omomo, Y., Sasaki, T., Wang, L. \& Watanabe, M. Redoxable nanosheet crystallites of $\mathrm{MnO}_{2}$ derived via delamination of a layered manganese oxide. J. Am. Chem. Soc. 125, 3568-3575 (2003).

18. Sun, Z. et al. Generalized self-assembly of scalable two-dimensional transition metal oxide nanosheets. Nat. Commun. 5, 3813 (2014).

19. Xiao, X. et al. Scalable salt-templated synthesis of two-dimensional transition metal oxides. Nat. Commun. 7, 11296 (2016)

20. Aryasetiawan, F. \& Gunnarsson, O. The GW method. Rep. Prog. Phys. 61, 237-312 (1998)

21. Ataca, C., Şahin, H. \& Ciraci, S. Stable, single-layer $M X_{2}$ transition-metal oxides and dichalcogenides in a honeycomb-like structure. J. Phys. Chem. C. 116, 8983-8999 (2012).

22. Rasmussen, F. A. \& Thygesen, K. S. Computational 2D materials database: electronic structure of transition-metal dichalcogenides and oxides. J. Phys. Chem. C. 119, 13169-13183 (2015)

23. Kresse, G., Surnev, S., Ramsey, M. G. \& Netzer, F. P. First-principles calculations for $\mathrm{V}_{\mathrm{x}} \mathrm{O}_{\mathrm{y}}$ Grown on $\mathrm{Pd}(111)$. Surf. Sci. 492, 329-344 (2001).

24. Vittadini, A. \& Casarin, M. Ab Initio modeling of $\mathrm{TiO}_{2}$ nanosheets. Theor. Chem. Acc 120, 551-556 (2008)

25. Dudarev, S. L., Botton, G. A., Savrasov, S. Y., Humphreys, C. J. \& Sutton, A. P. Electron-energy-loss spectra and the structural stability of nickel oxide: an LSDA +U study. Phys. Rev. B 57, 1505-1509 (1998).

26. Krukau, A. V., Vydrov, O. A., Izmaylov, A. F. \& Scuseria, G. E. Influence of the exchange screening parameter on the performance of screened hybrid functionals. J. Chem. Phys. 125, 224106 (2006).
27. Kan, M., Zhou, J., Sun, Q., Kawazoe, Y. \& Jena, P. The intrinsic ferromagnetism in a $\mathrm{MnO}_{2}$ monolayer. J. Phys. Chem. Lett. 4, 3382-3386 (2013).

28. Kan, E. et al. Two-dimensional hexagonal transition-metal oxide for spintronics. J. Phys. Chem. Lett. 4, 1120-1125 (2013).

29. Wang, J. \& Zhang, Y. Topologic connection between 2-D layered structures and 3-D diamond structures for conventional semiconductors. Sci. Rep. 6, 24660 (2016).

30. Boukhvalov, D. W. Stable antiferromagnetic graphone. Phys. E 43, 199-201 (2010).

31. Kresse, G. \& Hafner, J. Ab initio molecular-dynamics simulation of the liquidmetal-amorphous-semiconductor transition in germanium. Phys. Rev. B 49, 14251-14269 (1994).

32. Kresse, G. \& Furthmüller, J. Efficiency of Ab-Initio total energy calculations for metals and semiconductors using a plane-wave basis set. Comput. Mater. Sci. 6 , 15-50 (1996)

33. Kresse, G. \& Furthmüller, J. Efficient iterative schemes for $A b$ Initio total-energy calculations using a plane-wave basis set. Phys. Rev. B 54, 11169-11186 (1996).

34. Blöchl, P. E. Projector Augmented-Wave method. Phys. Rev. B 50, 17953-17979 (1994).

35. Kresse, G. \& Joubert, D. From ultrasoft pseudopotentials to the projector Augmented-Wave method. Phys. Rev. B 59, 1758-1775 (1999).

36. Perdew, J. P., Burke, K. \& Ernzerhof, M. Generalized gradient approximation made simple. Phys. Rev. Lett. 77, 3865-3868 (1996).

37. Perdew, J. P., Burke, K. \& Ernzerhof, M. Generalized gradient approximation made simple - errata. Phys. Rev. Lett. 78, 1396 (1997).

38. Bader, R. F. W. Atoms in Molecules: A Quantum Theory. (Oxford University Press, Oxford, 1990).

39. Henkelman, G., Arnaldsson, A. \& Jónsson, H. A fast and robust algorithm for Bader decomposition of charge density. Comput. Mater. Sci. 36, 354-360 (2006).

40. Yu, M. \& Trinkle, D. R. Accurate and efficient algorithm for Bader charge integration. J. Chem. Phys. 134, 064111 (2011)

41. Karlický, F., Zbořil, R. \& Otyepka, M. Band gaps and structural properties of graphene halides and their derivates: a hybrid functional study with localized orbital basis sets. J. Chem. Phys. 137, 034709 (2012).

42. Komsa, H.-P. \& Krasheninnikov, A. V. Native defects in bulk and monolayer $\mathrm{MoS}_{2}$ from first principles. Phys. Rev. B 91, 125304 (2015).

43. Mackrodt, W. C., Middlemiss, D. S. \& Owens, T. G. Hybrid density functional theory study of vanadium monoxide. Phys. Rev. B 69, 115119 (2004).

44. Franchini, C., Podloucky, R., Paier, J., Marsman, M. \& Kresse, G. Ground-state properties of multivalent manganese oxides: density functional and hybrid density functional calculations. Phys. Rev. B 75, 195128 (2007).

45. Schrön, A., Rödl, C. \& Bechstedt, F. Energetic stability and magnetic properties of $\mathrm{MnO}$ in the rocksalt, wurtzite, and zinc-blende structures: influence of exchange and correlation. Phys. Rev. B 82, 165109 (2010).

46. Guo, Y., Clark, S. J. \& Robertson, J. Electronic and magnetic properties of $\mathrm{Ti}_{2} \mathrm{O}_{3}$ $\mathrm{Cr}_{2} \mathrm{O}_{3}$, and $\mathrm{Fe}_{2} \mathrm{O}_{3}$ calculated by the screened exchange hybrid density functional. J. Phys.: Condens. Matter 24, 325504 (2012).

47. Zhu, L., Zhou, J., Guo, Z. \& Sun, Z. Metal-metal bonding stabilized ground state structure of early transition metal monoxide $\mathrm{TM}-\mathrm{MO}(\mathrm{TM}=\mathrm{Ti}, \mathrm{Hf}, \mathrm{V}, \mathrm{Ta})$. J. Phys. Chem. C. 120, 10009-10014 (2016).

48. Fang, C. M., Van Huis, M. A., Sluiter, M. H. F. \& Zandbergen, H. W. Stability, structure and electronic properties of $\mathrm{y}^{-} \mathrm{Fe}_{23} \mathrm{C}_{6}$ from first-principles theory. Acta Mater. 58, 2968-2977 (2010).

49. Nosé, S. A. Unified formulation of the constant temperature molecular dynamics methods. J. Chem. Phys. 81, 511-519 (1984)

50. Nosé, S. Constant temperature molecular dynamics methods. Prog. Theor. Phys. Suppl. 103, 1-46 (1991).

51. Bylander, D. M. \& Kleinman, L. Energy fluctuations induced by the Nosé thermostat. Phys. Rev. B 46, 13756-13761 (1992)

52. Bersuker, I. B. Pseudo-Jahn-Teller Effect-a two-state paradigm in formation deformation, and transformation of molecular systems and solids. Chem. Rev. 113, 1351-1390 (2013).

53. Jose, D. \& Datta, A. Understanding of the buckling distortions in silicene. J. Phys. Chem. C. 116, 24639-24648 (2012).

54. Nijamudheen, A., Bhattacharjee, R., Choudhury, S. \& Datta, A. Electronic and chemical properties of germanene: the crucial role of buckling. J. Phys. Chem. C. 119, 3802-3809 (2015).

55. Wang, Y. \& Ding, Y. Unexpected buckled structures and tunable electronic properties in arsenic nanosheets: insights from first-principles calculations. J. Phys.: Condens. Matter 27, 225304 (2015).

56. Wang, J., Meng, J., Li, Q. \& Yang, J. Single-layer cadmium chalcogenides: promising visible-light driven photocatalysts for water splitting. Phys. Chem. Chem Phys. 18, 17029-17036 (2016).

57. Revard, B. C., Tipton, W. W., Yesypenko, A. \& Hennig, R. G. Grand-canonical evolutionary algorithm for the prediction of two-dimensional materials. Phys. Rev. B 93, 054117 (2016). 
58. Singh, A. K., Mathew, K., Zhuang, H. L. \& Hennig, R. G. Computational screening of 2D materials for photocatalysis. J. Phys. Chem. Lett. 6, 1087-1098 (2015).

59. Shang, S.-L. et al. Lateral versus vertical growth of two-dimensional layered transition-metal dichalcogenides: thermodynamic insight into $\mathrm{MoS}_{2}$. Nano Lett. 16, 5742-5750 (2016).

60. Fu, B., Ge, Y., Su, W., Guo, W. \& Liu, C.-C. A new kind of 2D topological insulators BiCN with a giant gap and its substrate effects. Sci. Rep. 6, 30003 (2016).

61. De Groot, R. A., Mueller, F. M., Van Engen, P. G. \& Buschow, K. H. J. New class of materials: half-metallic ferromagnets. Phys. Rev. Lett. 50, 2024-2027 (1983).

62. Fang, C. M., De Wijs, G. A. \& De Groot, R. A. Spin-polarization in half-metals. J. Appl. Phys. 91, 8340-8344 (2002).

63. Katsnelson, M. I. et al. Half-metallic ferromagnets: from band structure to manybody effects. Rev. Mod. Phys. 80, 315-378 (2008).

64. Tomić, M., Jeschke, H. O. \& Valentí, R. Unfolding of electronic structure through induced representations of space groups: application to Fe-based superconductors. Phys. Rev. B 90, 195121 (2014).
65. Momma, K. \& Izumi, F. VESTA 3 for three-dimensional visualization of crystal, volumetric and morphology data. J. Appl. Crystallogr. 44, 1272-1276 (2011).

(i) Open Access This article is licensed under a Creative Commons Attribution 4.0 International License, which permits use, sharing, adaptation, distribution and reproduction in any medium or format, as long as you give appropriate credit to the original author(s) and the source, provide a link to the Creative Commons license, and indicate if changes were made. The images or other third party material in this article are included in the article's Creative Commons license, unless indicated otherwise in a credit line to the material. If material is not included in the article's Creative Commons license and your intended use is not permitted by statutory regulation or exceeds the permitted use, you will need to obtain permission directly from the copyright holder. To view a copy of this license, visit http://creativecommons. org/licenses/by/4.0/.

(c) The Author(s) 2019 